\title{
PLASTIC DEFORMATION MICROMECHANISM IN NANOTWINNED FILMS
}

\author{
N.V. Skiba ${ }^{1,2}$ \\ ${ }^{1}$ Institute of Problems of Mechanical Engineering, Russian Academy of Sciences, \\ St. Petersburg 199178, Russia \\ ${ }^{2}$ Peter the Great St. Petersburg State Polytechnic University, St. Petersburg 195251, Russia
}

Received: April 28, 2018

\begin{abstract}
A theoretical model is suggested which describes micromechanism of the plastic deformation in nanotwinned metallic film formed by electrical deposition onto metallic substrate. In the framework of the model, the micromechanism of the plastic deformation is widening of nanoscale twins due to migration of twin boundaries. The energy and stress characteristics of the twin widening are calculated.
\end{abstract}

\section{INTRODUCTION}

anostructured solids often possess unique mechanical characteristics such as high hardness, strength, and wear resistance [1-3]. Most nanocrystalline and ultrafine-grained material manifest low plasticity, which is undesirable for practical applications [13]. However, there are novel materials exhibiting simultaneously high strength and good ductility at room temperature; see, e.g., [4-12]. For example, it was revealed in experimental studies $[4,5]$ that nanotwinned cooper films (ultrafine-grained metallic films with high-density ensembles of nanoscale twins within grains) which were formed by electrical deposition demonstrate high strength with conservation of the functional plastic properties. According to the recent experimental $[4,5]$ and theoretical [13-16] works, the main micromechanism responsible for the unique combination of high strength and plasticity of nanotwinned materials is viewed to be plastic deformation occurring through widening of nanoscale twins due to migration of twin boundaries. In theoretical models [14-16] dislocation reactions resulting in formation of the twinning partial dislocations that move along the twin boundaries and provide widening of twins in bulk nanotwinned materials were considered. The main aim of this paper is to suggest a theoretical model which describes plastic deformation through widening of nanoscale twins in nanotwinned films with ultrafinegrained structure.

\section{MODEL OF NANOTWIN WIDENING}

Consider a two-dimensional model of an ultrafinegrained film with a periodic nanotwinned structure which was formed by electrical deposition onto a semi-infinite substrate with the same chemical composition. In the model, the film and the substrate are assumed to be elastically isotropic solids with the same shear modulus $G$ and Poisson's ratios n, and the same crystal lattice parameters $a$. Thus, the film-substrate interface does not create mismatch stresses. It is assumed that the grains of the nanotwinned film with an average size $d$ are composed of rectangular nanotwins bounded by coherent twin boundaries and grain boundaries (Fig. 1a). The action of the applied tensile load creates a re- 


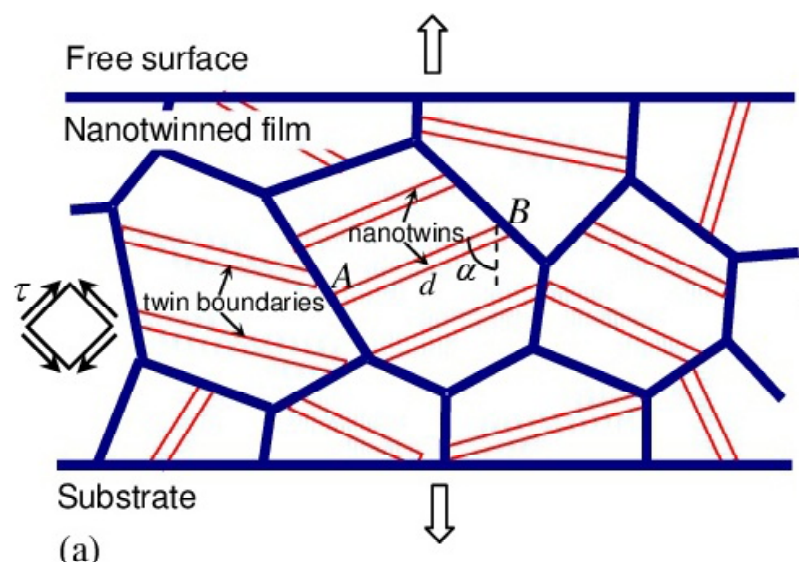

(a)

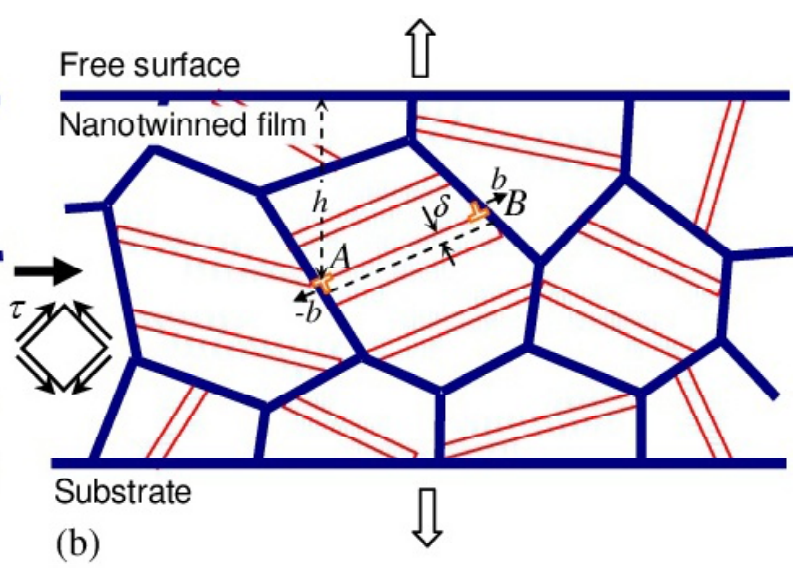

(b)

Fig. 1. A model of plastic deformation of an ultrafine-grained film with nanotwinned structure (with ultrafinegrained grains containing high-density ensembles of nanoscale twins). (a) A nanotwinned film specimen is under tensile load. (b) An elementary act of widening of nanotwin occurring through migration of a twin boundary $A B$ in the result of emission of a partial $b$-dislocation and its motion across the grain over twin boundary $A B$.

solved shear stress $\tau$ at the twin boundaries. If the resolved shear stress $\tau$ is high enough, it can lead to the emission of a partial dislocation with the Burgers vector $\mathbf{b}$ (partial $b$-dislocation) and its motion across the grain over a twin boundary. As the result, a dipole of the partial dislocations $A B$ with the Burgers vectors $\pm \mathbf{b}$ is formed at the junctions of the twin and grain boundaries (Fig. 1b). At the same time, slip of the partial dislocation over a coherent twin boundary $A B$ across the grain leads to the movement of this twin boundary $A B$ in the direction normal to the twin plane by one interplane distance $\delta$ providing the twin widening (Fig. 1b). The most works on the nanotwinned metals are concerned with copper [4,5,13-16]. So, in the framework of the model, we will consider widening of twins in fcc metallic films, that is, films with face-centered cubic crystal lattice. In fcc metals twin boundaries occupy the $\{111\}$ planes. Thus, we consider edge partial dislocations of $(a / 6)<11 \overline{2}>$ type as carriers of the dislocation slip in slip planes $\{111\}$ along twin boundaries. Such partial dislocations (Shockley dislocations) are characterized by the Burgers vector magnitude $b=a / \sqrt{6}$, where $a$ is the crystal lattice parameter. The distance $\delta$ between neighboring crystallographic slip planes $\{111\}$ in fcc metals equals $\delta=a / \sqrt{3}$. In the next session examine the energy characteristics of the process of the twin widening.

\section{ENERGY CHARACTERISTICS OF NANOTWIN WIDENING}

Consider the energy difference associated with the twin boundary migration (Fig. 1b). The event of twin boundary migration is characterized by the energy difference $\Delta W=W_{1}-W_{0}$, where $W_{1}$ and $W_{0}$ are the energy of the defect system after and before the twin boundary migration, respectively. The realization of the twin boundary migration event is energetically favored if $\Delta W<0$. The energy difference $\Delta W$ is given as:

$\Delta W=E_{s}^{d}-E_{\tau}$,

where $E_{s}^{d}$ is the proper energy of partial dislocation dipole $A B$ and $E_{\tau}$ is the work of the resolved shear stress $\tau$ on migration of the twin boundary by the distance $\delta$.

The proper energy $E_{s}^{d}$ can be derived from the stress function of an edge dislocation located near a free surface [17] and follows as

$$
\begin{aligned}
& E_{s}^{\alpha}=\frac{D b^{2}}{2}\left(\ln \frac{s^{2}+\left(h+h^{\prime}\right)^{2}}{p^{2}}+\right. \\
& \ln \frac{4 h h^{\prime}}{b^{2}}+\frac{4 h h^{\prime}}{s^{2}+\left(h+h^{\prime}\right)^{2}} \times \\
& \left.\left(\frac{s^{2}-\left(h+h^{\prime}\right)^{2}}{s^{2}+\left(h+h^{\prime}\right)^{2}}+2 \sin ^{2} \alpha \cos 2 \alpha\right)-1\right),
\end{aligned}
$$

where $D=G / 2 \pi(1-v), h^{\prime}=h-p \cos \alpha, s=p \sin \alpha, \alpha$ is the angle between twin boundary plane and normal to the free surface (Fig. 1a), $p$ is the distance traveled by the partial $b$-dislocation.

The work $E_{\tau}$ of the resolved shear stress $E_{\tau}$ on migration of the twin boundary by the distance $\delta$ follows as

$$
E_{\tau}=\tau b p \sin 2 \alpha .
$$



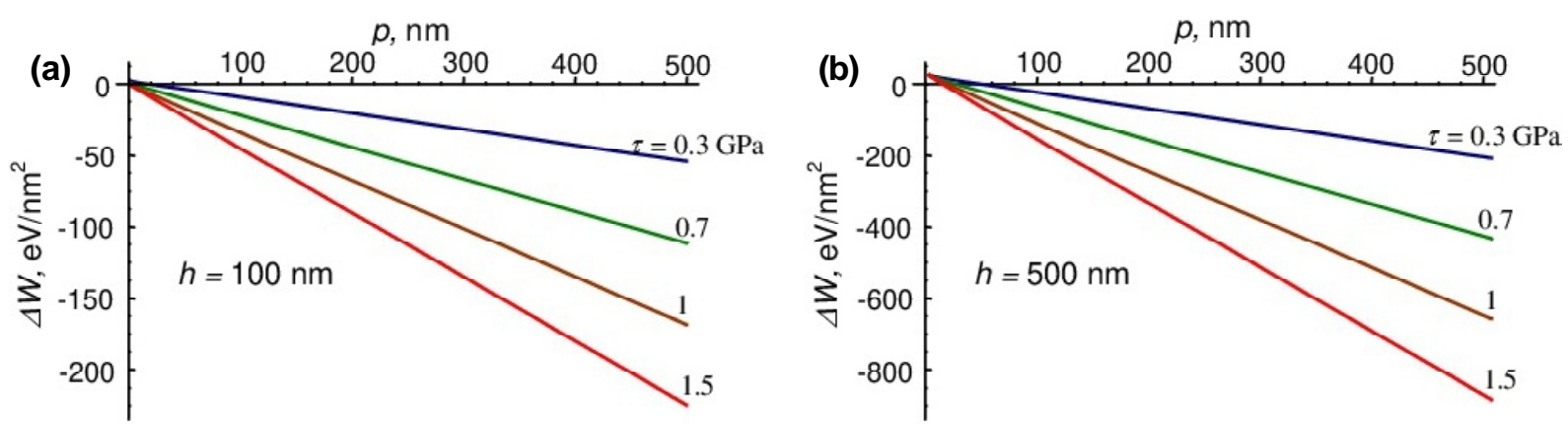

Fig. 2. Dependence of the energy difference $\Delta W$ on the distance $p$ travelled by the partial $b$-dislocation, at various values of the shear stress $\tau$ and, for the distance $h=100 \mathrm{~nm}$ (a) and $500 \mathrm{~nm}$ (b).

Formulas (1)-(3) allow us to calculate the energy difference $\Delta W$ characterizing the elementary act of plastic deformation occurring through widening of nanoscale twin. Using expression for the energy difference $\Delta W$, consider how $\Delta W$ changes when the partial $b$-dislocation slip along the twin boundary $A B$ (Fig. 1b). Let us perform such calculations in the exemplary case of a nanotwinned copper film characterized the following values of parameters: $G=44 \mathrm{GPa}, v=0.38, a=0.352 \mathrm{~nm}[18], d=500 \mathrm{~nm}$, and $\alpha=45^{\circ}$. The dependences of $\Delta W(d)$ are presented in Fig. 2, at various values of the shear stress $\tau$ and distance $h=100 \mathrm{~nm}$ (Fig. 2a) and $500 \mathrm{~nm}$ (Fig. $2 \mathrm{~b})$ between the nanotwin and the free surface. As it follows from Fig. 2, the dependences $\Delta W(d)$ monotonously decrease when the distance $p$ traveled by the partial $b$-dislocation increases. The dependences $\Delta W(d)$ presented in Fig. 2 are indicative the fact that the nanotwin widening becomes energetically favorable $(\Delta W(d)$ is negative and monotonously decreases) when the external stress $\tau$ reaches some critical value $\tau_{c} \approx 0.7 \mathrm{GPa}$, in the case $h=100 \mathrm{~nm}$ (Fig. 2a). Also, as it is seen from Fig. 2, when the distance $h$ between the nanotwin and the free surface increases, the process of the nanotwin widening is hampered.

\section{CONCLUSIONS}

Thus, in this paper, theoretical model has been developed that describes the micromechanism of the plastic deformation in ultrafine-grained metallic films with nanotwinned structure. In the framework of the model, the deformation mechanism in nanotwinned films represents the widening of the nanoscale twins due to the migration of the twin boundaries under the action of the mechanical load. It was shown that the nanotwin widening is energetically favorable in certain ranges of parameters of deformed metallic films with nanotwinned structure. Our theoretical estimate of the value of the critical stress at which the process of the nanotwin widening is energetically favorable is rather well consistent with the experimentally measured $[4,5]$ values of the flow stresses in nanotwinned copper films.

\section{ACKNOWLEDGEMENTS}

This work was supported by the Russian Fund of Basic Research (grant 16-32-60110) and Russian Ministry of Education and Science (task 16.3483.2017/PCh).

\section{REFERENCES}

[1] C.C. Koch, I.A. Ovid'ko, S. Seal and S. Veprek, Structural Nanocrystalline Materials: Fundamentals and Applications (Cambridge, Cambridge University Press, 2007).

[2] H.A. Padilla II and B.L. Boyce // Exp. Mechanics 50 (2010) 5.

[3] Y. Estrin and A. Vinogradov // Acta Mater. 61 (2013) 782.

[4] L. Lu, X. Chen, X. Huang and K. Lu // Science 323 (2009) 607.

[5] K. Lu, L. Lu and S. Suresh // Science 324 (2009) 349.

[6] I.-C. Choi, Y.-J. Kim, Y.M. Wang, U. Ramamurty and J.-I. Jang // Acta Mater. 61 (2013) 7313.

[7] J.R. Greer // Nature Mater. 12 (2013) 689.

[8] Y.M. Wang, F. Sansoz, T. LaGrange, R.T. Ott, J. Marian, T.W. Barbee, Jr and Alex V. Hamza // Nature Mater. 12 (2013) 697.

[9] Z. Zhang, Y. Huo, F. Huo, X. Zhang, L. Zhang and D. Guo // Scr. Mater. 68 (2013) 747.

[10] Z. Zhang, F. Li, G. Ma, R. Kang and X. Guo // Scr. Mater. 69 (2013) 231. 
[11] K.Y. Yu, D. Bufford, F. Khatkhatay, H. Wang, M.A. Kirk and X. Zhang // Scr. Mater. 69 (2013) 385.

[12] Z. You, X. Li, L. Gui, Q. Lu, T. Zhu, H. Gao and L. Lu // Acta Mater. 61 (2013) 217.

[13] N.V. Morozov, I.A. Ovid'ko and N.V. Skiba // Rev. Adv. Mater. Sci. 37 (2014) 29.

[14] N.F. Morozov, I.A. Ovid'ko and N.V. Skiba // Doklady Physics 60 (2015) 507.

[15] I.A. Ovid'ko, A.G. Sheinerman and N.V. Skiba // Rev. Adv. Mater. Sci. 41 (2015) 93.
[16] N.V. Skiba, I.A. Ovid'ko, A.G. Sheinerman and Ya.V. Konakov // Mater. Phys.Mech. 30 (2017) 40.

[17] A.E. Romanov and V.I. Vladimorov, Dislocation in Solids. vol. 9, ed F R N Nabarro (Amsterdam: North-Holland, 1992), p. 191.

[18] J.P. Hirth and J. Lothe, Theory of Dislocations (Wiley, New York, 1982). 